\title{
Mit der Seife verschwindet das Händchen
}

\author{
Cristina Regazzi
}

A lte Menschen hüten Grosskinder, sind Omas oder Opas, sitzen auf Bänken und beobachten das an ihnen vorbeigehende Leben, backen Kuchen, gehen mit Hündchen spazieren oder wandern. Als städtische Alternative spazieren sie in aller Ruhe zwischen der gestressten «working people» und besetzen Zug- und Tramsitze in der Rush-Hour und zählen ihr Kleingeld an der Supermarktkasse kurz vor Ladenschluss. So will es noch manches Klischée, so halten sich hartnäckige Altersbilder.

Doch ältere Menschen sind heute mehr denn je nicht nur physisch und geistig fit, sondern auch offen gegenüber neuen Entwicklungen. Dies - entgegen anders lautenden Vorurteilen - durchaus auch gegenüber den sich rasant entwickelnden Technologien, die unser Leben (meistens) bereichern.

Smartphone, Tablet und Computer registrieren in der Altersgruppe $65+$ bedeutende und weiterhin wachsende Verkaufszahlen. Dementsprechend wird auch im Alter gesurft, geskyped und getouched. Aber schon wer heute eine neue Waschmaschine kauft, muss im digitalen Bereich sattelfest sein: Entweder kann man die Bedienungsmenus intuitiv benutzen oder man schlägt sich mit dicken Bedienungsanleitungen herum. Auch der neue Steamer in der Küche bietet tolle Rezepte auf Knopfdruck - vorausgesetzt man weiss, wie das Ding funktioniert. Selbst die Armaturenbretter in unseren Autos sehen vermehrt aus wie Flugzeugcockpits oder Oberflächen von Videogames.

Technologien, die unser Alltagsleben unterstützen, sogenannte Ambient/Active Assisted Living (AAL)-Technologien, werden präsenter und immer günstiger. Sie zielen auf die Unterstützung der Selbstständigkeit und das Wohnen zu Hause für ältere Menschen, und glaubt man der Industrie oder den Erfahrungen aus Japan, so werden Roboter zukünftig in der Pflege vermehrt Fuss fassen.
Kein Wunder wächst die Anzahl von Kursangeboten für Senioren auch im Bereich Informationstechnologien. Auch meine Mama (80) hat nach langem Zögern vor einigen Jahren an einem PC-Kurs teilgenommen: zuerst mit grossem Respekt, dann mit grossem Spass. Besonderes Gelächter hat es ausgelöst, als sie irgendwann etwas genervt gerufen hat: «Aaaah! Wenn ich die Seife da bewege, verschwindet das Händchen!» (Sie hat die Mouse herumgeschüttelt und den Cursor am Bildschirm aus der Sicht verloren.) Heute sucht sie selbstverständlich Informationen und Rezepte online, googelt die Feriendestinationen ihrer Töchter und verschickt Nachrichten mit oder ohne Fotos von ihrem Handy. Das aktuelle Computer-Modell hat sie vor einem Jahr gekauft - und dabei besonderen Wert auf ein stylisches Design gelegt!

Alter und Technik? Natürlich! Sinnvollerweise sollten aber schon bei der Konzeption und beim Design der Produkte oder Dienstleistungen die Bedürfnisse von alternden Menschen berücksichtigt werden. Denn diese (anspruchsvoller werdende) Klientel hat Bedürfnisse, welche oft denen jüngerer Menschen gleichen, denn auch diese möchten keine 250-seitige Bedienungsanleitung für die neue Waschmaschine lesen!

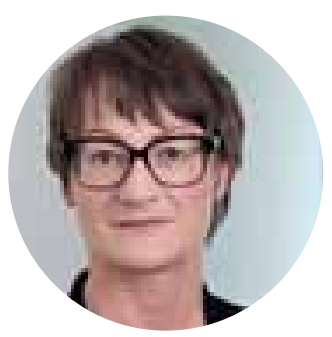

Cristina Regazzi Gerontologin Hochschule Luzern (HSLU), Leiterin AZ (Richterwil/Samstagern), Beratung Marketing/Kommunikation.

c.regazzi@bluewin.ch 


\title{
« L’espèce de savon fait disparaître la petite main »
}

\author{
Cristina Regazzi
}

L

es personnes âgées gardent les petits-enfants, sont des mamies ou des papis, s'asseyent sur des bancs et regardent la vie passer, font des gâteaux, vont se promener avec les chiens ou font de la randonnée. Contrairement aux autres citadins, ils se promènent tranquillement parmi les « travailleurs » stressés, occupent les places assises dans les trains et les trams pendant l'heure de pointe et comptent leur monnaie à la caisse des supermarchés juste avant la fermeture. Ce sont là de nombreux clichés tenaces sur les personnes âgées.

Cependant elles sont aujourd'hui plus que jamais non seulement en forme physiquement et mentalement, mais également souvent ouvertes aux nouvelles évolutions et ceci, contrairement à des préjugés discordants, face aux technologies à l'évolution fulgurante qui enrichissent (le plus souvent) notre vie.

Smartphone, tablette et ordinateur enregistrent d'importants chiffres de vente sans cesse croissants dans la tranche d'âge des plus de 65 ans. En conséquence, les personnes âgées surfent sur Internet, utilisent Skype ainsi que la fonction tactile. Déjà quand on achète une machine à laver aujourd'hui, on doit bien s'y connaître en numérique : soit on arrive à utiliser de manière intuitive le menu d'exploitation soit on se débat avec un épais manuel de l'utilisateur. Même le nouveau cuiseur vapeur dans la cuisine propose de superbes recettes rien qu'en appuyant sur un bouton, à condition de savoir comment marche ce machin. Et que dire des tableaux de bord de nos voitures qui ressemblent plus à des cockpits d'avion ou à des surfaces de jeux vidéo!

Les technologies qui nous assistent dans notre vie quotidienne, les technologies «Ambient/Active Assisted Living $(A A L)$ », sont de plus en plus présentes et de plus en plus avantageuses. Elles visent à favoriser l'autonomie et la vie à la maison pour les personnes âgées et, si l'on en croit l'industrie ou l'expérience japonaise, les robots prendront de plus en plus pied dans le domaine des soins à l'avenir.

Pas étonnant que le nombre d'offres de cours pour seniors augmente également dans le domaine des technologies de l'information. Même ma maman (80 ans), après avoir longuement hésité, a participé à un cours d'informatique il y a de cela quelques années : d'abord avec un grand respect, puis avec un grand plaisir. Elle déclenchait les rires lorsqu'elle s'énervait et criait parfois : «Aaaah!» «Si je bouge l'espèce de savon là, la petite main disparaît! » (elle a secoué la souris et a perdu de vue le curseur sur l'écran). Aujourd'hui, elle cherche bien entendu des informations et des recettes en ligne, utilise Google pour les destinations de vacances de sa fille et envoie des messages avec ou sans photo de son téléphone portable. Son modèle actuel d'ordinateur, elle l'a acheté il y a un an et tenait particulièrement à un design stylé !

Technologie et vieillesse ? Naturellement ! Mais les besoins des personnes vieillissantes doivent être pris en compte intelligemment lors de la conception et du design des produits et des prestations. Car cette clientèle (de plus en plus exigeante) a des besoins qui ressemblent souvent à ceux des personnes plus jeunes car ces derniers également ne souhaitent pas lire le manuel de 250 pages de la nouvelle machine à laver !

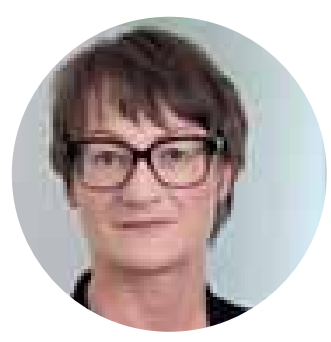

Cristina Regazzi Gérontologue Hochschule Luzern (HSLU), directrice centre pour personnes agées (Richterswil/Samstagern), experte en marketing/communication

c.regazzi@bluewin.ch 